\title{
A daily herd Markov-chain model to study the reproductive and economic impact of reproductive programs combining timed artificial insemination and estrus detection
}

\author{
J. O. Giordano, ${ }^{1}$ A. S. Kalantari, P. M. Fricke, M. C. Wiltbank, and V. E. Cabrera ${ }^{2}$ \\ Department of Dairy Science, University of Wisconsin-Madison 53706
}

\begin{abstract}
Our objective was to compare the economic and reproductive performance of programs combining timed artificial insemination (TAI) and different levels of AI after estrus detection (ED) using a daily Markov-chain model. A dairy herd was modeled with every cow following daily probabilistic events of aging, replacement, mortality, pregnancy, pregnancy loss, and calving. The probability of pregnancy depended on the combination of probability of insemination and conception rate (CR). All nonpregnant cows had a probability of pregnancy between the end of the voluntary waiting period and days in milk cutoff for AI. After the cutoff, cows were labeled as do not breed and replaced when milk production was below a minimum milk threshold. A similar model was created to represent a replacement heifer herd to simulate and adjust the supply and demand of replacements. The net value (NV) of a program was the sum of milk income over feed cost, replacement and mortality cost, income from newborns, and reproductive costs. The model was used to compare the NV of 19 programs. One program used 100\% TAI (42\% CR for first TAI and 30\% for second-and-later services), whereas the other programs combined TAI with ED. The proportion of cows receiving AI after ED for the combined programs ranged from 30 to $80 \%$, with levels of $\mathrm{CR}$ of 25,30 , and $35 \%$. As the proportion of cows receiving AI after ED increased, the CR of cows receiving TAI decreased. The combined programs with CR of $35 \%$ for cows receiving AI after ED had the greatest NV and reproductive performance at all levels of ED. The program using 100\% TAI had greater NV and better reproductive performance than all programs with $25 \%$ CR after ED inseminations, whereas it had very similar performance to combined programs with up to $60 \%$ of cows receiving AI after ED and $30 \%$ CR.
\end{abstract}

\footnotetext{
Received September 23, 2011.

Accepted April 27, 2012.

${ }^{1}$ Current address: Department of Animal Science, Cornell University, Ithaca, NY 14853.

${ }^{2}$ Corresponding author: vcabrera@wisc.edu
}

The factor with the greatest relative contribution to the differences among programs was income over feed cost, followed by replacement and reproductive costs. Adjusting the days in milk cutoff for AI to match the supply and demand of heifer replacements improved the NV of all programs except for those with $25 \%$ CR after ED, which had either no change or a decrease in NV. In summary, the economic value of reproductive management programs combining TAI and ED depended on the proportion of cows receiving $\mathrm{AI}$ after ED and the resulting CR. Adjusting the heifer supply and demand increased the NV of programs with heifer surplus and decreased the NV of programs with heifer deficit.

Key words: herd simulation, reproduction decision support system, reproductive performance economics, reproductive program comparison

\section{INTRODUCTION}

Dairy herd profitability is closely associated with reproductive performance (Louca and Legates, 1968; Britt, 1985; Meadows et al., 2005; Giordano et al., 2011). Optimizing the time that adult cows spend in the most efficient part of the lactation curve (Pecsok et al., 1994; Ferguson and Galligan, 1999), minimizing replacement due to reproductive failure (Pecsok et al., 1994; Giordano et al., 2011), and maximizing the number of female calves born for replacements affects dairy farm revenue. Furthermore, all of these factors depend on the outcome of the reproductive program (Louca and Legates, 1968; Pecsok et al., 1994; Meadows et al., 2005; Giordano et al., 2011). Numerous reproductive management strategies are available for dairy farms (Pursley et al., 1995; Moreira et al., 2001; Fricke et al., 2003; Souza et al., 2008); however, due to the complex interactions among multiple biological and management factors affecting dairy herd dynamics and economics, it remains a challenge for dairy producers to determine the best management program to implement.

In recent years, the dairy industry has adopted the use of hormonal protocols for synchronization of estrus and ovulation as reproductive management tools to improve the overall reproductive efficiency of lactating 
dairy cows (Caraviello et al., 2006; Olynk and Wolf, 2008). Protocols such as Ovsynch (Pursley et al., 1995) are useful to increase insemination risk but not fertility in lactating dairy cows, as the conception rate (CR) of AI services after Ovsynch is usually within the range observed for cows inseminated after a detected estrus (Pursley et al., 1997; Rabiee et al., 2005). More recently, new protocols such as Presynch-Ovsynch (Moreira et al., 2001), G6G (Bello et al., 2006), and Double-Ovsynch (Souza et al., 2008) were developed to increase not only the insemination risk but also the fertility of timed AI (TAI). Frequently, US commercial dairy herds use one of these protocols for insemination of cows for the first service postpartum, followed by the use of the Ovsynch protocol for resynchronization of ovulation for second and subsequent AI services. Some dairies rely solely on synchronization for insemination and perform 100\% TAI without estrus detection (ED), whereas the majority implement a combined program using TAI and estrus detection. In this case, the same protocols are used; however, only cows not detected in estrus before or during the protocol will receive a TAI. Under field conditions, it is difficult to determine the optimal balance between cows receiving AI after a detected estrus, their CR, and the CR of cows reaching the TAI. The impact of these multiple interactions on the overall profitability of the dairy operation is usually difficult to predict. As a consequence, simulation models that replicate the dynamics of a dairy herd are useful tools to aid producers and consultants in the decision-making process.

Numerous simulation models have been used to predict the impact of a wide range of reproductive management scenarios and performance levels on the profitability of dairy operations (Schmidt, 1989; Plaizier et al., 1997; De Vries, 2004, 2006; Groenendaal et al., 2004; Meadows et al., 2005; Olynk and Wolf, 2009; Giordano et al., 2011). These models have included different levels of complexity to replicate the sequence of events in the productive cycle of dairy cows. In this regard, the UW-DairyRepro\$ model was recently developed based on an event driven simulation model using Markov chains and partial budgeting (Giordano et al., 2011) to compare the future profitability of a dairy herd when using different reproductive management schemes. That model is a reliable tool to simulate the economic and reproductive performance of dairy herds; however, to remain user friendly, such a model was built with some specific limitations. For example, groups of cows are not followed through successive lactations; cows not pregnant leave the herd at the cutoff DIM for insemination and are replaced regardless of their milk production; pregnancy losses are not taken into account; and AI after ED occurs during $1 \mathrm{~d}$ only after the end of the voluntary waiting period ( VWP) or a previous AI service. As a result of these limitations, the previous model may overlook some differences in reproductive dynamics and economic outcomes observed with the use of different management programs on dairy farms. Consequently, a new daily Markov-chain model was developed to perform a more detailed assessment of the dynamics of reproductive programs using novel simulation approaches.

The objectives of this study were (1) to create a daily simulation model using a Markov-chain framework to simulate the reproductive, productive, and economic dynamics of dairy herds; (2) to use this daily model to assess the economic and reproductive outcomes of adding varying levels of estrus detection with 3 different outcomes for CR to a $100 \%$ TAI program using Presynch-Ovsynch for first service and Ovsynch for resynchronization of ovulation in lactating dairy cows; and (3) to study the economic implications of a replacement strategy focused on matching the supply and demand of heifers for each reproductive program.

\section{MATERIALS AND METHODS}

\section{Simulation Model for Adult Cows}

A Markov-chain model (St-Pierre and Jones, 2001; De Vries, 2004; Cabrera et al., 2006) was developed and used to simulate a dairy herd and assess the economic value of distinct reproductive programs. A Markovchain model was defined by cow states, stages, and transition probabilities. The cow states describe the characteristics of the cows in the model, stages define the time between 2 sequential events, and transition probabilities determine the cow's flow among states of parity, DIM, and reproductive status from one stage to the next. The model used 3 cow states that included parity $(l=1$ to 9$)$, DIM $(D I M=1$ to 750$)$ and reproductive status $(p=0$ to 282 , where $p=0$ denoted nonpregnant cows and $p=1$ to 282 denoted days in pregnancy). To gain precision and accuracy in the calculations and to better represent reality, the stage length of the model was set to $1 \mathrm{~d}$. The use of a daily Markov-chain model enables precise monitoring of the impact of reproductive programs as they likely happen in commercial dairy herds.

Every cow in the model followed daily probabilistic events of aging, replacement, dying, getting pregnant, aborting, calving, and starting a new lactation. A defined lactation curve determined milk production, which depended on parity, DIM, and pregnancy status. The herd structure was defined by transition probabili- 
ties of (1) replacement due to nonreproductive reasons, (2) death, (3) reproductive performance, and (4) pregnancy loss. Also, the herd structure was affected by replacement due to reproductive failure based upon a predefined DIM cutoff for AI and milk yield threshold. A large algorithm was used to estimate iteratively the proportion of cows in every cow state defined in the model. The model included approximately 600,000 possible cow states, depending on the herd dynamics generated by each reproductive program simulated. A solution to the problem was found when the herd population reached a steady state. Steady state of the herd population occurred when the number of cows in each specific state (parity, DIM, and pregnancy status) no longer changed from one iteration to the next.

Transition probabilities defined the probabilities of a group of cows moving among defined cow states. For instance, a nonpregnant cow could become pregnant, be replaced, or die and a pregnant cow could abort, be replaced, die, or calve at the end of gestation. These events occurred daily for each cow in the herd. Transition matrices of involuntary replacement, mortality, pregnancy, and pregnancy loss were defined as daily probabilities following the model dimensions.

The proportion of cows in each state in the next stage or next day $\left(P s_{l, D I M+1, p}\right)$ was calculated as follows: for nonreplaced cows, breeding eligible, AI, and becoming pregnant:

$P s_{l, D I M+1,1}=P s_{l, D I M, 0} \times\left(1-\right.$ Pleave $\left._{l, D I M}\right) \times$ Ppreg $_{l, D I M} ;$

for nonreplaced cows, breeding eligible, AI, and not becoming pregnant:

$$
\begin{aligned}
P s_{l, D I M+1,0}= & \text { Ps }_{l, \text { DIM }, 0} \times\left(1-\text { Pleave }_{l, D I M}\right) \\
& \times\left(1-\text { Ppreg }_{l, \text { DIM }}\right) ;
\end{aligned}
$$

for nonreplaced cows and not breeding eligible:

$$
P s_{l, D I M+1,0}=P s_{l, D I M, 0} \times\left(1-\text { Pleave }_{l, D I M}\right) ;
$$

for pregnant cows and not aborting:

$$
\begin{aligned}
P s_{l, D I M+1, p+1} & =P s_{l, D I M, p} \times\left[\left(1-\text { Pleave }_{l, p}\right)\right. \\
& \left.\times\left(1-\text { PAbort }_{p}\right)\right] ;
\end{aligned}
$$

for pregnant cows and aborting:

$$
P s_{l, D I M+1,0}=P s_{l, D I M, p} \times\left[\left(1-\text { PleaveP }_{l, p}\right) \times\left(\text { PAbort }_{p}\right)\right] ;
$$

for cows calving and moving to the next parity:

$$
\begin{gathered}
P s_{l+1,1,0}=\sum_{\text {DIM }=V W P+\text { gest }}^{\text {repcull }+ \text { gest }} P s_{l+1,1,0} \\
+\left[P s_{l, D I M, 282} \times\left(1-\text { Pleave }_{l, 282}\right)\right],
\end{gathered}
$$

where $l=$ parity and $p=$ pregnancy; Pleave $O_{l, D I M}$ and Pleave $P_{l, p}$ are the daily transition probabilities of leaving (nonreproductive and reproductive replacement, and mortality) of nonpregnant and pregnant cows, respectively; Ppreg $_{D I M}$ is the daily transition probability of pregnancy; PAbort $_{p}$ is the daily transition probability of pregnancy loss; repcull is the cutoff DIM for breeding in days; $V W P$ is the voluntary waiting period in days; and gest is the gestation length in days. To maintain a constant herd size (Meadows et al., 2005; De Vries, 2006), cows leaving the herd at any stage or day were replaced at the beginning of the next stage or day as $P s_{1,1,0}$ (Figure 1).

After the model reached steady state, the net economic value (NV; $\$ /$ cow per day) of a given reproductive program $(r)$ was calculated as the sum product of

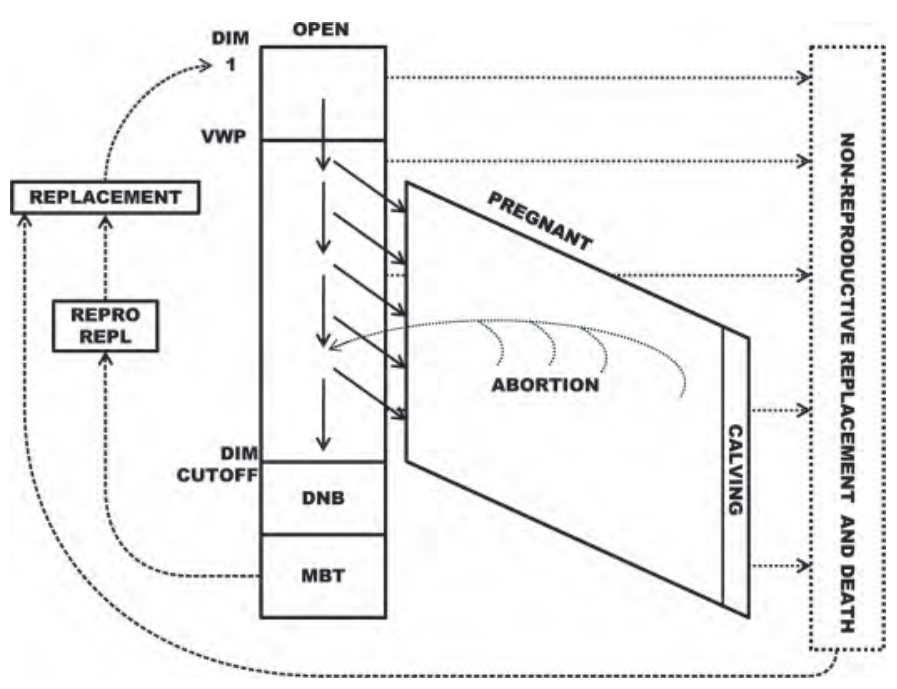

Figure 1. Schematic representation of the herd dynamics in the daily Markov-chain model. Cows enter the lactating herd after calving and begin to transition into lactation in daily steps until the end of the voluntary waiting period (VWP). From the end of the VWP until the DIM cutoff for insemination, cows have a daily probability of pregnancy. Cows failing to become pregnant are coded as do not breed (DNB) and remain in the herd until milk production falls below a predefined threshold (milk below threshold, MBT) when they are replaced (Repro Repl). Cows conceiving after AI transition into the pregnant stream and progress throughout the entire gestation until calving occurs at 282 d. Pregnant cows have a daily probability of pregnancy loss from 30 to $281 \mathrm{~d}$ in gestation. Cows suffering pregnancy loss return to the nonpregnant cow stream. Every day throughout lactation, cows have a daily probability of replacement due to nonreproductive reasons and death. All cows leaving the herd for any reason are replaced the next day with a first-lactation cow. DIM cutoff = maximum number of DIM for insemination. 
the proportion of cows in each state of the model by the economic factors of milk income over feed cost (IOFC), income from newborn calves $(C V)$, nonreproductive replacement cost $(N R R C)$, mortality cost $(M C)$, reproductive replacement cost $(R R C)$, and the reproductive program cost $(R P C)$. Different reproductive programs yield different herd structures and, consequently, different NV:

$$
\begin{gathered}
N V_{r}=\sum_{l=1}^{9} \sum_{D I M=1}^{750} \sum_{p=0}^{282} P s_{l, D I M, p} \\
\times(I O F C+C V+N R R C+M C+R R C+R P C)_{l, D I M, p} .
\end{gathered}
$$

\section{Simulation Model for Heifers}

Another daily simulation model was used to quantify the heifer supply of each reproductive program. The female calves born remaining alive for $48 \mathrm{~h}$ from the adult cow simulation model were accumulated over all lactations. These calves then were simulated in a similar fashion as the adult cows until first calving. Transition probabilities of mortality and reproductive performance were then used to project the number of heifers available as replacements. Additional details of this model are provided in subsequent sections.

\section{Calculation of Bioeconomic Simulation Variables}

Income Over Feed Cost Calculations. The IOFC for each cow state was calculated by subtracting the cost of feeding from the milk production value at each cow state.

Milk Production. The MilkBot model (Ehrlich, 2011) was used to fit farm-observed lactation curves. The MilkBot model predicts milk production $(\boldsymbol{M P})$ based on 4 parameters: scale $(a), \operatorname{ramp}(b)$, offset $(c)$, and decay ( $d$; for more details, please refer to http:// milkbot.com):

$$
M P_{D I M}=a \times\left(1-\frac{e^{\left(\frac{c-D I M}{b}\right)}}{2}\right) \times e^{-d \times D I M} .
$$

A nonlinear optimization (Levenberg-Marquardt algorithm; Press et al., 2007) was used to fit the MilkBot parameters to observed milk tests. This fitting algorithm minimized the residuals between observed (dairy farm records) and predicted (MilkBot model) data points.
DMI. The DMI ( $\mathrm{kg} / \mathrm{cow}$ per day) was a function of maintenance and milk production according to $\mathrm{BW}$ $(\mathrm{kg})$ and $4 \%$ FCM being produced, according to NRC (2001):

$$
\begin{aligned}
D M I_{D I M}= & \left(0.372 \times F C M_{D I M}+0.0968 \times B W^{0.75}\right) \\
& \times\left(1-e^{\left.-0.192 \times\left[\left(\frac{D I M}{7}\right)+3.67\right]\right) .}\right.
\end{aligned}
$$

$\boldsymbol{B} \boldsymbol{W}$. The BW was calculated for each cow state using the Korver function as described by van Arendonk (1985) as a cumulative function of the age of a cow (according to parity), DIM, and pregnancy status using the parameters described by Kalantari et al. (2010).

\section{Calf Value Calculations}

The value added by calves born from pregnant cows was calculated as the market value of the proportion of male calves, female calves, and discounting stillbirths. The male-to-female calf ratio was set at 53.3:46.7 as reported in Silva del Río et al. (2007).

\section{Replacement and Mortality Cost Calculations}

The total cost of replacement and mortality was calculated by adding the cost of cows being replaced due to nonreproductive reasons, dying, and replaced due to reproductive failure. Replacement due to reproductive failure occurred when cows failed to become pregnant by the time of a predefined DIM cutoff for AI services and when milk production was below a minimum milk threshold. Cows not pregnant at the DIM cutoff and still producing above the minimum milk production threshold were labeled as do not breed and remained in the herd until their milk production fell below the minimum threshold. The cost of replacement or death for each cow state was calculated as the product of the probability of replacement or probability of death and their associated costs. The associated cost for nonreproductive and reproductive replacement was similarly estimated as the heifer replacement cost, which was partially compensated by the value of the calf coming with the replacement heifer and by the salvage value of the replaced cow. The salvage value of a replaced cow was calculated as the product of the estimated BW of the cow leaving the herd and the market value of beef in dollars per kilogram. The cost of mortality was estimated as the heifer replacement cost, which was 
only compensated by the value of the calf coming with the replacement heifer.

The model included a daily hazard risk for any cow, depending on DIM and parity. In addition, the model distinguished hazard risks for nonpregnant and pregnant cows. Daily hazard risks for nonreproductive replacement and death were calculated based on De Vries et al. (2010) who reported a greater risk of cows leaving the herd in early lactation, late lactation, and in later parities (parities 1 to 5 and $\geq 6$ ). Probability of nonreproductive replacement and death were summarized in daily vectors with the dimensions of the model described here. Mortality rates were calculated as a proportion $(17 \%)$ of these hazard risks according to DHI benchmark data (AgSource Cooperative Services, 2011). The hazard risks for pregnant cows were set at $25 \%$ of the hazard risks of nonpregnant cows (De Vries et al., 2010).

The number of nonreproductive replaced and dead cows during a stage $(1 \mathrm{~d})$ was calculated as the product of the number of cows in each state in the model and their probability of replacement or death. Cows calving in their last parity (lactation 9) were assumed to be at the end of their productive life and were, therefore, replaced (St-Pierre and Jones, 2001; Cabrera, 2010). The implication of the latter assumption is minimal, because under most circumstances $<1 \%$ of the herd population reaches those states (Cabrera and Giordano, 2010).

\section{Reproductive Program Cost Calculations}

Total reproductive cost for a specific program resulted from the aggregation of 5 economic factors: labor for estrus detection, hormones for synchronization of ovulation, labor for hormone injections, AI, and pregnancy diagnosis.

Labor for Estrus Detection. The cost of labor for ED (\$/cow per d) was calculated by dividing the daily cost of ED by the number of cows available for ED on that day. The daily cost of ED was calculated by multiplying the number of personnel involved in daily ED, by the number of hours per day those personnel were performing the task and by the cost per hour of labor. The approximate number of available cows for ED was estimated by multiplying the total number of lactating cows by the number of effective days of ED, which were obtained by dividing $1 \mathrm{yr}$ by the length of the calving interval.

Hormones for Synchronization. The cost of each hormone injection and its associated labor cost $(\$ /$ cow per day) were charged only to the proportion of cows eligible to receive such treatment based on the reproductive program, reproductive status (nonpregnant cows eligible for AI or pregnant cows with unknown status at the time of resynchronization), and DIM.

Labor for Hormone Injections. The labor cost of administering hormones (\$/cow per day) was calculated by dividing the daily cost of administering hormones by the number of cows available for such treatment on a day. The daily cost of administering hormones was calculated by multiplying the number of personnel by the number of hours required to administer the injections by the cost per hour of labor.

$\boldsymbol{A I}$. The costs of AI, which included the cost of semen and the labor cost to perform an insemination, were charged only to those cows that were inseminated after a detected estrus or after completion of the synchronization protocol according to the defined reproductive program, reproductive status, and DIM.

Pregnancy Diagnosis. The cost of performing a pregnancy diagnosis (\$/cow per day) was calculated by dividing the daily cost of pregnancy diagnosis by the number of cows being diagnosed on a day. The daily cost of pregnancy diagnosis was calculated by multiplying the number of personnel involved (e.g., veterinarian or technician) by the number of hours required by the cost per hour of labor. The cost of performing the first pregnancy diagnosis postinsemination was charged to all cows that failed to conceive after the TAI for the pure TAI program and only to those cows not detected in estrus in between 2 inseminations (in both groups of cows, determination of pregnancy status was required to complete the protocol) for programs combining TAI and ED. For cows becoming pregnant after AI, pregnancy diagnosis costs were charged at the specific day in gestation that the test was performed. The same cost of pregnancy diagnosis was applied to cows remaining pregnant and not aborting in subsequent pregnancy reconfirmations.

\section{Herd Reproductive Performance Simulation}

The model can accommodate reproductive programs relying completely on ED, TAI, or combinations of both strategies. For a pure ED program, the VWP concluded at the DIM at which cows detected in estrus were eligible to be inseminated, whereas for a pure TAI program the end of the VWP coincided with the DIM at the first TAI service. For a program combining ED and TAI for first service postpartum, the VWP defined the DIM at which cows detected in estrus were eligible to be inseminated.

Cows not pregnant after first service postpartum were inseminated either after ED or TAI according to the program used. When TAI was used for second and subsequent AI services of all or only those cows failing 
to be detected in estrus, cows received AI at a defined interval after the previous AI, depending on the time required to complete the resynchronization of ovulation protocol. The time interval between 2 resynchronized TAI was defined as the interbreeding interval, which was a function of the number of days required to complete the different resynchronization protocols. All nonpregnant cows in a herd could be inseminated and, therefore, had a probability of becoming pregnant any day between the end of the VWP and the DIM cutoff for insemination. The DIM cutoff at which nonpregnant cows were no longer inseminated was set to a predefined DIM (e.g., 300 DIM), depending on the reproductive program. The daily probability of pregnancy $(\boldsymbol{D P P})$ depended on the reproductive program under consideration and was calculated as a separated dimension to the matrix that simulated the herd structure. The specific proportion of cows receiving AI, not pregnant, and becoming pregnant was calculated at each DIM between the end of the VWP and the cutoff DIM for reproductive services. The proportion of pregnant cows (DPP) was then incorporated to the matrix that simulated the herd structure transitioning to the first day in gestation and was subtracted from the pool of nonpregnant cows.

For a pure ED program the DPP depended upon the probability of cows being detected in estrus and inseminated (service rate, $\boldsymbol{S R}$ ) and the probability of conceiving or conception rate $(C R E D)$ after each AI. Nonpregnant cows after an AI service were eligible for reinsemination during the next cycle:

$$
D P P_{D I M}=S R_{D I M} \times C R E D .
$$

For pure TAI programs, the daily probability of pregnancy depended on the SR and the CR of TAI services (CRTAI). Service rate for TAI was $100 \%$ of nonpregnant cows present at the TAI:

$$
D P P_{D I M}=S R_{D I M} \times C R T A I .
$$

When a program combined ED with TAI, daily probabilities for pregnancy were calculated similar to those for the pure ED and TAI programs, except that breeding-eligible cows for TAI were those not pregnant after the previous TAI and not detected in estrus during the period following the AI service. Cows inseminated in estrus in the cycle preceding the current TAI were only eligible for reinsemination during the following cycle or TAI service attempt. Therefore, after each TAI service, all of the cows not pregnant to the TAI and those inseminated and not pregnant during the previous ED cycle were eligible for insemination.
The probability of a cow being detected in estrus and receiving an $\mathrm{AI}$ in programs combining $\mathrm{ED}$ and TAI was not uniform throughout the breeding cycle. When cows had their estrous cycle synchronized or during the cycle following a TAI service, the distribution of cows showing estrus followed a particular pattern. For example, after the $2 \mathrm{PGF}_{2 \alpha}$ injections of a Presynch program, most cows would show estrous behavior and would receive AI within 3 to $7 \mathrm{~d}$ with the maximum frequency around $3 \mathrm{~d}$ (Chebel et al., 2006). After a TAI, most cows would be detected in estrus and would receive AI between 18 to $27 \mathrm{~d}$ after TAI with the maximum frequency around $22 \mathrm{~d}$ (Bartolome et al., 2005). To simulate similar patterns of estrous behavior, the distribution of cows receiving AI after ED after the 2 $\mathrm{PGF}_{2 \alpha}$ injections of Presynch as reported by Chebel et al. (2006) and the distribution of cows inseminated after a previous TAI as reported by Bartolome et al. (2005) were fitted through polynomial and exponential equations. To fit the distribution of cows receiving AI in estrus after the second $\mathrm{PGF}_{2 \alpha}$ injection of Presynch, 2 polynomial equations were used as follows:

$$
\begin{gathered}
\mathrm{F}=0.088 \mathrm{D}^{2}-0.247 \mathrm{D}+0.214 \\
\text { (for } \mathrm{D}=1 \text { to } 3 \text { after the } \mathrm{PGF}_{2 \alpha} \text { injection); } \\
\mathrm{F}=0.002 \mathrm{D}^{2}-0.049 \mathrm{D}+0.323 \\
\text { (for } \mathrm{D}=4 \text { to } 11 \text { after the } \mathrm{PGF}_{2 \alpha} \text { injection), }
\end{gathered}
$$

where $\mathrm{F}=$ fitted value for percentage of cows inseminated on each day (\%) and $\mathrm{D}=$ day after $\mathrm{PGF}_{2 \alpha}$ injection of Presynch. The total percentage of eligible cows after the second $\mathrm{PGF}_{2 \alpha}$ of Presynch was then multiplied by the fitted value for each day (from $\mathrm{D}=1$ to 11 ) to obtain the actual percentage of cows inseminated on each day.

To fit the distribution of cows receiving AI in estrus after each TAI, $1 \%$ of the cows were distributed for each day from $\mathrm{d} 1$ to 17 after TAI and then the percentage of cows AI from d 18 to 31 after TAI was fitted with a polynomial and an exponential equation as follows:

$$
\begin{gathered}
\mathrm{F}=0.005 \mathrm{D}^{2}-0.173 \mathrm{D}+1.534 \\
(\text { for } \mathrm{D}=18 \text { to } 22 \text { after } \mathrm{TAI}) \\
\mathrm{F}=169.74^{-0.316 \mathrm{D}}(\text { for } \mathrm{D}=23 \text { to } 31 \text { after TAI })
\end{gathered}
$$

where $\mathrm{F}=$ fitted value for percentage of cows inseminated on each day (\%) and D = day after TAI. The total percentage of eligible cows after a TAI service was then multiplied by the fitted value for each day (from 
$\mathrm{D}=1$ to 31 after TAI) to obtain the actual percentage of cows inseminated on each day.

\section{Probability of Pregnancy Loss}

Pregnant cows had a daily probability of losing their pregnancy from d 1 in gestation to term. Daily probabilities of pregnancy loss were defined for the transition matrix of pregnancy ( 1 to $282 \mathrm{~d}$ ). The number of cows that aborted in $1 \mathrm{~d}$ was calculated by multiplying the vectors: number of pregnant cows in each pregnancy state by the probability of pregnancy loss. Cows losing their pregnancy joined the nonpregnant cows in the next stage (Figure 1). The probability of pregnancy loss varied according to the stage of gestation. The probability of early embryonic loss up to $30 \mathrm{~d}$ in gestation was set at $0 \%$ because reported $\mathrm{CR}$ are considered to be approximations of the CR at the time of pregnancy diagnosis at approximately $30 \mathrm{~d}$ in gestation. The probability of pregnancy loss was then considered to be $12.5 \%$ from 30 to $45 \mathrm{~d}$ in gestation (Chebel et al., 2004), $9.9 \%$ from 46 to $180 \mathrm{~d}$ in gestation (Ettema and Santos, 2004), and 2\% from $181 \mathrm{~d}$ to term (P. M. Fricke and J. O. Giordano, unpublished data). These probabilities were converted to daily pregnancy loss risks to be incorporated in the model described here.

\section{Calculated Reproductive Performance Parameters}

The simulation model calculated reproductive parameters typically used to monitor reproductive performance of dairy herds. Calculations were based on the simulated herd dynamics generated with the reproductive performance data entered as input. Twenty-one day pregnancy rate (21-d $\mathbf{P R})$, which indicates the rate at which eligible cows become pregnant (Ferguson and Galligan, 1999), was calculated by dividing the total proportion of cows that became pregnant in each 21-d period between the end of the VWP and the DIM cutoff for AI services by that proportion of eligible cows at the beginning of each 21-d period. For this 21-d PR calculation, a cow was considered to be pregnant only when it reached $35 \mathrm{~d}$ in gestation. Additional parameters calculated by the model included average days open (DO), projected calving interval (actual interval between 2 calving events plus projected interval for nonpregnant cows, which was calculated by adding length of gestation to the DIM cutoff; Fetrow et al., 1990), and average DIM for the herd.

\section{Software Application}

The model was developed as a standalone executable program with Visual Basic.Net 2010 (Microsoft Corp.,
Seattle, WA). Input parameters of transition probabilities, economic variables, and reproductive programs are input as spreadsheet files. The model then runs iterations until reaching steady state. The number of iterations to reach steady state varies depending on the input parameters and defined reproductive programs, but, on average, requires about 5,000 iterations. The results are accessible as comma-separated files that can be loaded into text or spreadsheet programs. Each reproductive program detailed for the case study presented herein was defined with its respective parameters and entered as inputs in the model. The model then provided the total value of the reproductive program and its associated economic, productive, and reproductive herd statistics.

\section{Case Study: Assessing the Effect of Adding Different Levels of Estrus Detection and Conception Rate to a TAI Program}

Reproductive Programs. Nineteen reproductive programs were simulated (Table 1) to evaluate and compare the economic and reproductive performance when using a program that relies on $100 \%$ TAI for all AI services with programs that combine different levels of ED, which had 3 different levels of CR after ED inseminations. More specifically, program 1 relied completely on TAI for all services after synchronization of ovulation for first postpartum AI with the PresynchOvsynch protocol (Moreira et al., 2001), whereas the Ovsynch protocol (Pursley et al., 1995) was used for second and subsequent AI services. For program 1, the end of the VWP was set at 72 DIM coincident with the first service TAI. All cows received the first GnRH injection of the Ovsynch protocol for resynchronization of ovulation, regardless of their pregnancy status $32 \mathrm{~d}$ after TAI. Nonpregnancy diagnosis was performed $7 \mathrm{~d}$ later by rectal palpation and those cows not pregnant to the previous TAI service continued the protocol to receive their next TAI $42 \mathrm{~d}$ after the previous service.

For programs 2 to 19, the same synchronization of ovulation protocols were used except that AI after ED was added between the end of the VWP set at 50 DIM and the first TAI at 72 DIM and in between TAI services (ED was discontinued at the time of the first GnRH of the Ovsynch protocol for all AI services). The proportion of cows inseminated after ED was sequentially increased from 30 to $80 \%$ in 10-percentage-point increments (Table 1). Three groups of programs were created for which the CR after ED was 25 (programs 2 to 7 ), 30 (programs 8 to 13), and 35\% (programs 14 to 19). Under most circumstances, when ED is added before or between TAI services, a reduced CR is observed for those cows inseminated to TAI because the 
Table 1. Expected reproductive performance of programs used for simulation in the case study

\begin{tabular}{|c|c|c|c|c|c|c|c|}
\hline $\begin{array}{l}\text { Program } \\
\text { number }\end{array}$ & Program & \multicolumn{3}{|c|}{ First AI } & \multicolumn{3}{|c|}{ Second and subsequent AI } \\
\hline 2 & $\mathrm{TAI}+\mathrm{ED} 2^{4}$ & 30 & 25 & 40 & 30 & 25 & 30 \\
\hline 3 & TAI+ED 3 & 40 & 25 & 38 & 40 & 25 & 30 \\
\hline 7 & TAI+ED 7 & 80 & 25 & 30 & 80 & 25 & 28 \\
\hline 8 & TAI+ED 8 & 30 & 30 & 40 & 30 & 30 & 30 \\
\hline 9 & TAI+ED 9 & 40 & 30 & 38 & 40 & 30 & 30 \\
\hline 10 & TAI+ED 10 & 50 & 30 & 36 & 50 & 30 & 30 \\
\hline 11 & TAI+ED 11 & 60 & 30 & 34 & 60 & 30 & 28 \\
\hline 16 & $\mathrm{TAI}+\mathrm{ED} 16$ & 50 & 35 & 36 & 50 & 35 & 30 \\
\hline 17 & TAI+ED 17 & 60 & 35 & 34 & 60 & 35 & 28 \\
\hline 18 & TAI+ED 18 & 70 & 35 & 32 & 70 & 35 & 28 \\
\hline 19 & TAI+ED 19 & 80 & 35 & 30 & 80 & 35 & 28 \\
\hline
\end{tabular}

${ }^{1}$ Percentage of cows experiencing AI after estrus detection (ED) before first timed AI (TAI).

${ }^{2}$ Conception rate $(\mathrm{CR})$ of cows experiencing AI after ED.

${ }^{3}$ Program TAI 1 relied on 100\% TAI for all services after synchronization of ovulation for first postpartum AI with the Presynch-Ovsynch protocol, whereas the Ovsynch protocol was used for second and subsequent AI services. The voluntary waiting period (VWP) was $72 \mathrm{~d}$ and the interval between 2 successive inseminations was $42 \mathrm{~d}$.

${ }^{4}$ Programs combining TAI with estrus detection (TAI+ED 2 to 19) used the same synchronization of ovulation protocols as TAI except that AI after ED was added between the end of the VWP set at 50 DIM and the first TAI at 72 DIM and in between TAI services (ED was discontinued at the time of the first GnRH injection of the Ovsynch protocol for all AI services).

fertility potential of the population of cows reaching this point is different than when no estrus detection is added (Chebel et al., 2004; Keskin et al., 2011). Therefore, for each 10-percentage-point increment in ED before first service TAI from 30 to $80 \%$, the initial CR of $40 \%$ after TAI services decreased by 2 percentage points (Table 1). Additionally, the CR of second and subsequent TAI services also was decreased by 2 percentage points when the proportion of cows receiving AI after ED was between 60 to $80 \%$ (Table 1). The DIM cutoff for insemination was set at 300 DIM for all programs.

Reproductive Program Cost. Input parameters to calculate the cost of performing each of the reproductive programs was assumed to be for a 1,000-cow commercial herd in Wisconsin using a reproductive management program of similar characteristics to the one used in the case study. The cost of $\mathrm{PGF}_{2 \alpha}$ was set at $\$ 2.3 /$ dose, whereas the cost of $\mathrm{GnRH}$ at $\$ 2.6$ / dose according to the market value of 2 commercially available products in Wisconsin. The cost of semen and performing an insemination was set at $\$ 10$ per AI. The veterinary cost of performing pregnancy diagnosis by rectal palpation was set at $\$ 105$ per hour of labor. First pregnancy diagnosis for pregnant cows occurred at 39 d in gestation with 2 pregnancy reconfirmations at 67 and $221 \mathrm{~d}$ in gestation. Only those cows not pregnant to a previous AI that reached and completed the resynchronization of ovulation protocol to receive their next TAI (determination of pregnancy status was required to complete the protocol) were charged the cost of pregnancy diagnosis, whereas cows detected in estrus and AI received no charges (reinseminated before the day of pregnancy testing). Because the 19 simulated programs assumed that the resynchronization of ovulation protocol started $32 \mathrm{~d}$ after a previous AI when the reproductive status of cows was unknown, the cost of a GnRH injection was included for pregnant cows also.

Milk Production Fitting. Observed milk production records were collected from a large (1,000 lactating cows) commercial Holstein herd in Wisconsin and fitted with the MilkBot model as previously described (Figure 2). A factor for milk production depression because of gestation was used based on De Vries (2004) that indicated that MP would be reduced by 5,10 , and $15 \%$ in months of gestation 5, 6, and 7, respectively. These 

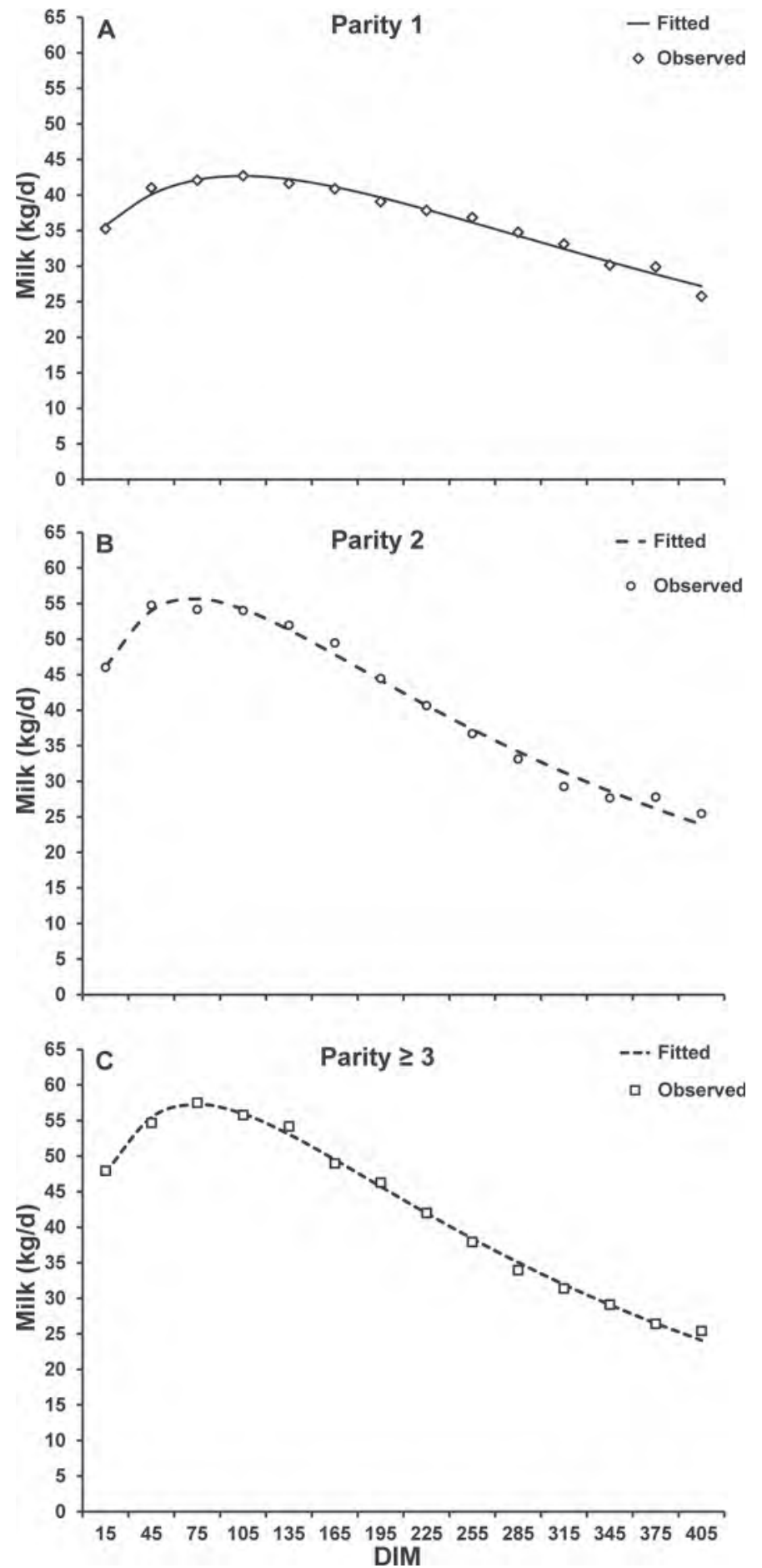

Figure 2. Lactation curves used for the case study simulations. Milk production information compiled from a commercial herd (dots) was fitted using the MilkBot model (dotted line; Ehrlich, 2011). Individual curves were fitted for cows in the first (panel A), second (panel B), and third and subsequent (panel C) parities to obtain daily milk production for the simulations. values were converted to daily factors to be incorporated into the model.

Price of Milk, Feed, Calves, Replacement, and Salvage Value. Input prices included $\$ 0.36 / \mathrm{kg}$ of milk; $\$ 0.17$ and $\$ 0.13 / \mathrm{kg}$ of feed (DM) for lactating and nonlactating cows, respectively; $\$ 136 /$ calf for females; $\$ 50 /$ calf for males; $\$ 1,302 /$ heifer replacement; and $\$ 1.16 / \mathrm{kg}$ for culled cows. All input variables were the average values reported in the Cost of Production bulletin published by the Center for Dairy Profitability at the University of Wisconsin-Madison (http://cdp. wisc.edu/) for the period of April 2010 to April 2011.

Reproductive Programs for Replacement Heifers. All females born on the farm entered the heifer replacement matrix and began the rearing process. The probability of death was $6.5 \%$ from calving to weaning and $1.8 \%$ from weaning to calving based on data from the US Department of Agriculture (USDA, 2010). In addition, heifers had a daily probability of pregnancy from 400 to $670 \mathrm{~d}$ of age. Inseminations were performed after detection of estrus only with no synchronization being used. The SR across all AI services was set at $65 \%$, whereas the CR was set at $53 \%$ for first and $51 \%$ for second and subsequent AI services according to Kuhn et al. (2006).

\section{Changing the Cutoff DIM for Insemination to Balance Heifer Supply and Demand}

Under most circumstances, the total number of heifers in the stream of replacements that reach calving and the number of heifers required by the lactating herd to maintain a constant herd size will differ according to the reproductive dynamics of the simulated herd. A potential strategy to balance heifer supply and demand is then to change the DIM cutoff for insemination, which in turn, will change the total number of opportunities for cows to become pregnant and the replacement pattern at the end of the breeding period. For the case study, once the lactating herd reached steady state and the total number of heifers required to maintain a constant herd size was known, attempts were made to balance supply and demand of heifers. The original 300 DIM cutoff for insemination was either reduced or increased until the closest value to zero (either positive or negative) for heifer balance was obtained.

\section{RESULTS}

\section{Net Value Differences for the Programs Compared}

All results presented herein were obtained from the different modules comprising the model and represent 
Table 2. Total net value (NV; \$/cow per year) and relative contribution of milk income over feed cost (IOFC), replacement cost, reproductive program cost, and calf value to the total NV of each of the programs simulated for the case study

\begin{tabular}{lccccc}
\hline Program & IOFC & $\begin{array}{c}\text { Replacement } \\
\text { cost }\end{array}$ & $\begin{array}{c}\text { Reproductive } \\
\text { cost }\end{array}$ & Calf value & Total NV \\
\hline TAI 1 & $3,362.8$ & 184.2 & 41.3 & 42.0 & $3,179.3$ \\
& & & & & \\
TAI+ED 2 & $3,357.9$ & 187.8 & 39.0 & 40.2 & $3,171.4$ \\
TAI+ED 3 & $3,355.7$ & 189.2 & 38.1 & 39.4 & $3,167.9$ \\
TAI+ED 4 & $3,354.4$ & 190.5 & 36.9 & 38.8 & $3,165.8$ \\
TAI+ED 5 & $3,352.9$ & 193.0 & 35.7 & 37.6 & $3,161.9$ \\
TAI+ED 6 & $3,353.1$ & 193.9 & 33.9 & 37.3 & $3,162.6$ \\
TAI+ED 7 & $3,353.7$ & 194.6 & 31.8 & 37.1 & $3,164.4$ \\
& & & & & \\
TAI+ED 8 & $3,362.1$ & 185.3 & 38.0 & 41.6 & $3,180.4$ \\
TAI+ED 9 & $3,361.6$ & 185.8 & 36.8 & 41.4 & $3,180.4$ \\
TAI+ED 10 & $3,361.6$ & 186.1 & 35.4 & 41.2 & $3,181.3$ \\
TAI+ED 11 & $3,361.6$ & 187.4 & 33.9 & 40.7 & $3,181.0$ \\
TAI+ED 12 & $3,362.9$ & 187.2 & 32.0 & 40.8 & $3,184.6$ \\
TAI+ED 13 & $3,364.9$ & 186.9 & 29.7 & 41.1 & $3,189.4$ \\
& & & & & \\
TAI+ED 14 & $3,366.3$ & 182.9 & 37.1 & 43.0 & $3,189.2$ \\
TAI+ED 15 & $3,367.2$ & 182.7 & 35.7 & 43.1 & $3,192.0$ \\
TAI+ED 16 & $3,368.6$ & 182.2 & 34.1 & 43.4 & $3,195.6$ \\
TAI+ED 17 & $3,369.9$ & 182.6 & 32.4 & 43.3 & $3,198.2$ \\
TAI+ED 18 & $3,372.5$ & 181.7 & 30.4 & 44.0 & $3,204.4$ \\
TAI+ED 19 & $3,375.6$ & 180.8 & 28.1 & 44.6 & $3,211.3$ \\
\hline
\end{tabular}

${ }^{1}$ Program timed AI (TAI) 1 relied on 100\% TAI for all services after synchronization of ovulation for first postpartum AI with the Presynch-Ovsynch protocol, whereas the Ovsynch protocol was used for the second and subsequent AI services. The voluntary waiting period (VWP) was $72 \mathrm{~d}$ and the interval between 2 successive inseminations was $42 \mathrm{~d}$.

${ }^{2}$ Programs combining TAI with estrus detection (TAI+ED 2 to 19), used the same synchronization of ovulation protocols as TAI except that AI after ED was added between the end of the VWP set at 50 DIM and the first TAI at 72 DIM and in between TAI services (ED was discontinued at the time of the first GnRH injection of the Ovsynch protocol for all AI services).

the simulated herd in steady state. The total NV $(\$ /$ cow per year) and the relative contribution of each of the 4 components of the NV are presented in Table 2. In addition, the NV differences between the 18 programs combining TAI and ED (programs 2 to 19) with the $100 \%$ TAI program (TAI 1) are presented in Figure 3. All programs having $25 \% \mathrm{CR}$ after ED breedings before first service TAI and between resynchronized TAI services had a lower NV than TAI 1. As the proportion of cows inseminated after ED increased from 30 to $60 \%$, the NV differences increased from $\$ 7.9$ to $\$ 17.4 /$ cow per year in favor of TAI 1. A decreasing trend in the NV differences was observed when the percentage of cows receiving AI after ED was 70 and $80 \%$; however, the NV differences remained within the $\$ 15$ cow/yr range. By contrast, for programs having $30 \% \mathrm{CR}$ to AI services after ED (8 to 13 ), the NV was always superior for the combined programs, with differences ranging from $\$ 1.1$ to $\$ 10.1 /$ cow per year in favor of the combined programs. Small differences were observed when the percentage of cows receiving AI after ED was between 30 to $60 \%$ (equal or less than $\$ 2 /$ cow per year), whereas greater differences on the order of $\$ 5$ and $\$ 10 /$ cow per year were observed when the percentage of cows receiving AI after ED was 70 and $80 \%$, respectively. Similarly, when the CR to AI services between TAI was $35 \%$, the NV differences were all positive, ranging from $\$ 9.9$ to $\$ 32.0 /$ cow per year in favor of the programs in which ED was added between TAI services. In this case, the NV differences with TAI 1 increased by every 10-percentage-point increment in the percentage of cows inseminated after ED to reach a maximum difference when the percentage of cows receiving $\mathrm{AI}$ after $\mathrm{ED}$ was $80 \%$.

A comparison between the absolute values of IOFC, replacement cost, reproductive program cost, and calf value indicated that the major contributor to the total $\mathrm{NV}$ in dollars per cow per year was the IOFC, which was $\$ 3362.4 /$ cow per year, on average, for the 19 programs, as opposed to $\$ 186.6, \$ 34.7$, and $\$ 41.1 /$ cow per year for replacement cost, reproductive program cost, and calf value, respectively (Table 2). Furthermore, when assessing the relative contribution to the differences among the 19 programs, IOFC accounted for an average difference of $\$ 7.8 /$ cow per year, with a range of $\$ 0.0$ to $\$ 22.7$ (Figure 4 ). By contrast, the average 


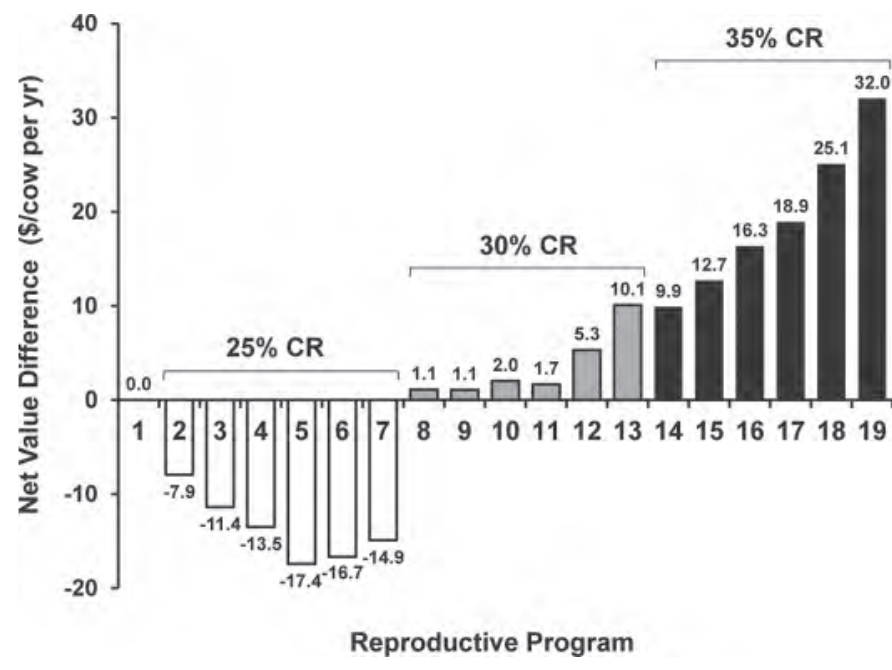

Figure 3. Difference in net value ( $\$$ /cow per year) for 18 programs combining estrus detection and timed AI with a $100 \%$ timed AI program as defined in Table 1 . $\mathrm{CR}=$ conception rate.

difference between programs due to replacement cost and reproductive program cost was similar in magnitude and variation, with $\$ 4.8$ (range $=\$ 0.04$ to $\$ 13.8$ ) and $\$ 4.0 /$ cow per year (range $=\$ 0.0$ to $\$ 13.2 /$ cow per year) for replacement and reproductive program costs, respectively (Figure 4). Furthermore, calf value had the lowest contribution to the difference, with $\$ 2.6 /$ cow per year and ranging between $\$ 0.04$ and $\$ 7.6 /$ cow per year (Figure 4).

\section{Reproductive Dynamics}

The calculated performance for the 19 reproductive programs based on the model simulations are presented in Figure 5. Twenty-one day pregnancy rate for a preset VWP of $50 \mathrm{~d}$ varied between 14 and $20 \%$. In general, those programs having $25 \% \mathrm{CR}$ to AI services after ED had the lowest 21-d PR, especially when the proportion of cows receiving AI after ED varied between 60 and $80 \%$. When the CR to AI after ED was 30\%, the 21-d PR fluctuated between 16 and $17 \%$, whereas for the combined programs having $35 \% \mathrm{CR}$ to AI after ED, the 21-d PR ranged between 18 to $20 \%$. For the latter, higher values were observed as the proportion of cows inseminated after ED increased to reach a maximum of 20\% 21-d PR when $80 \%$ of the cows were inseminated after a detected estrus. For TAI 1, the 21-d PR was $17 \%$ when calculated based on a 50-d VWP, whereas it was $20 \%$ when the VWP was set at 72 DIM, coincident with the time of the first postpartum TAI.

Average DO ranged between 120 to $131 \mathrm{~d}$ for the 19 studied programs. Although average DO for most

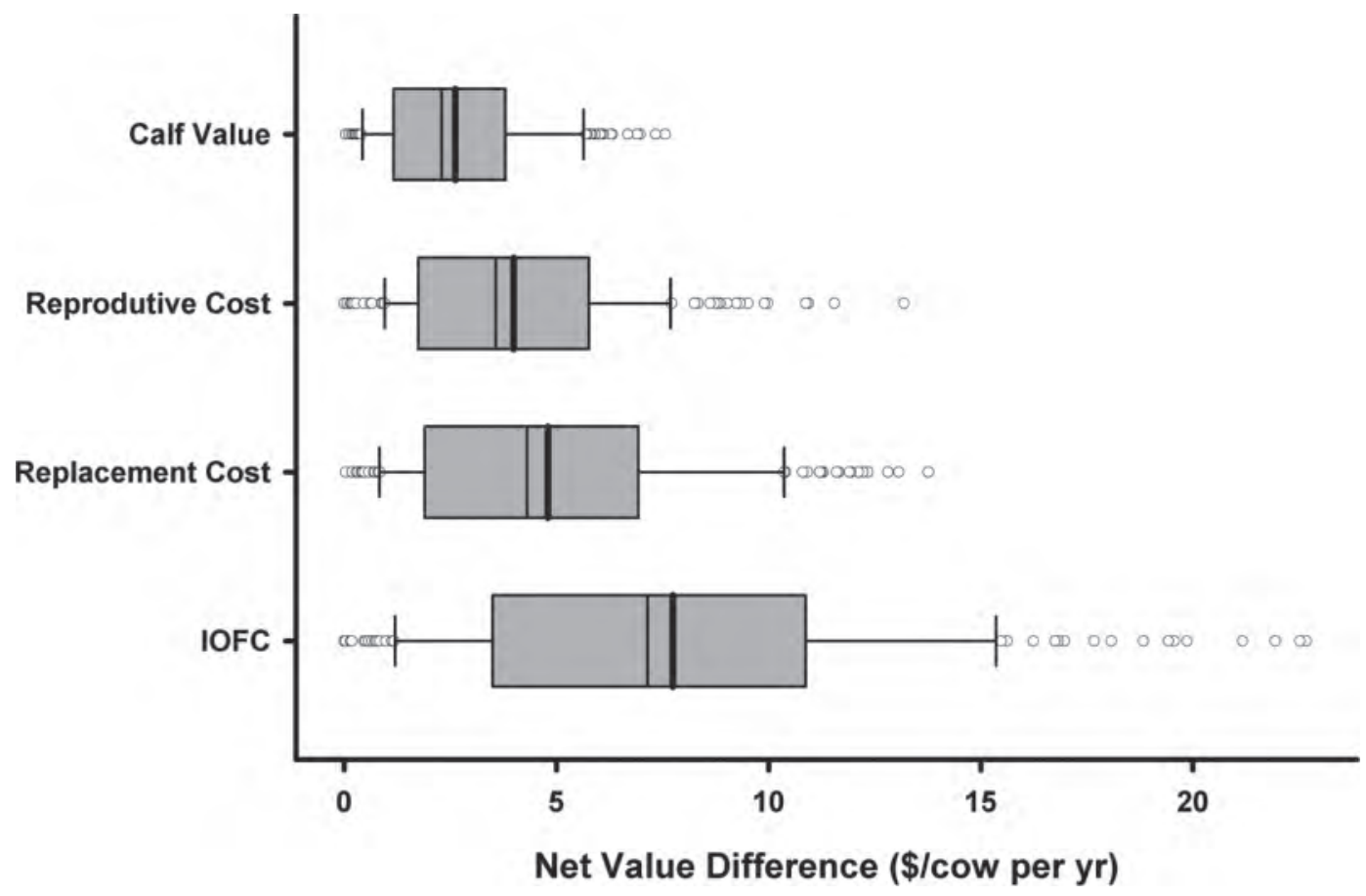

Figure 4. Box plot representing the individual contribution of income over feed cost (IOFC), replacement cost, reproductive program cost, and calf value to the total net value $(\$ /$ cow per year) differences between the 19 programs compared in the case study. Solid bold line inside box represents the mean difference. 

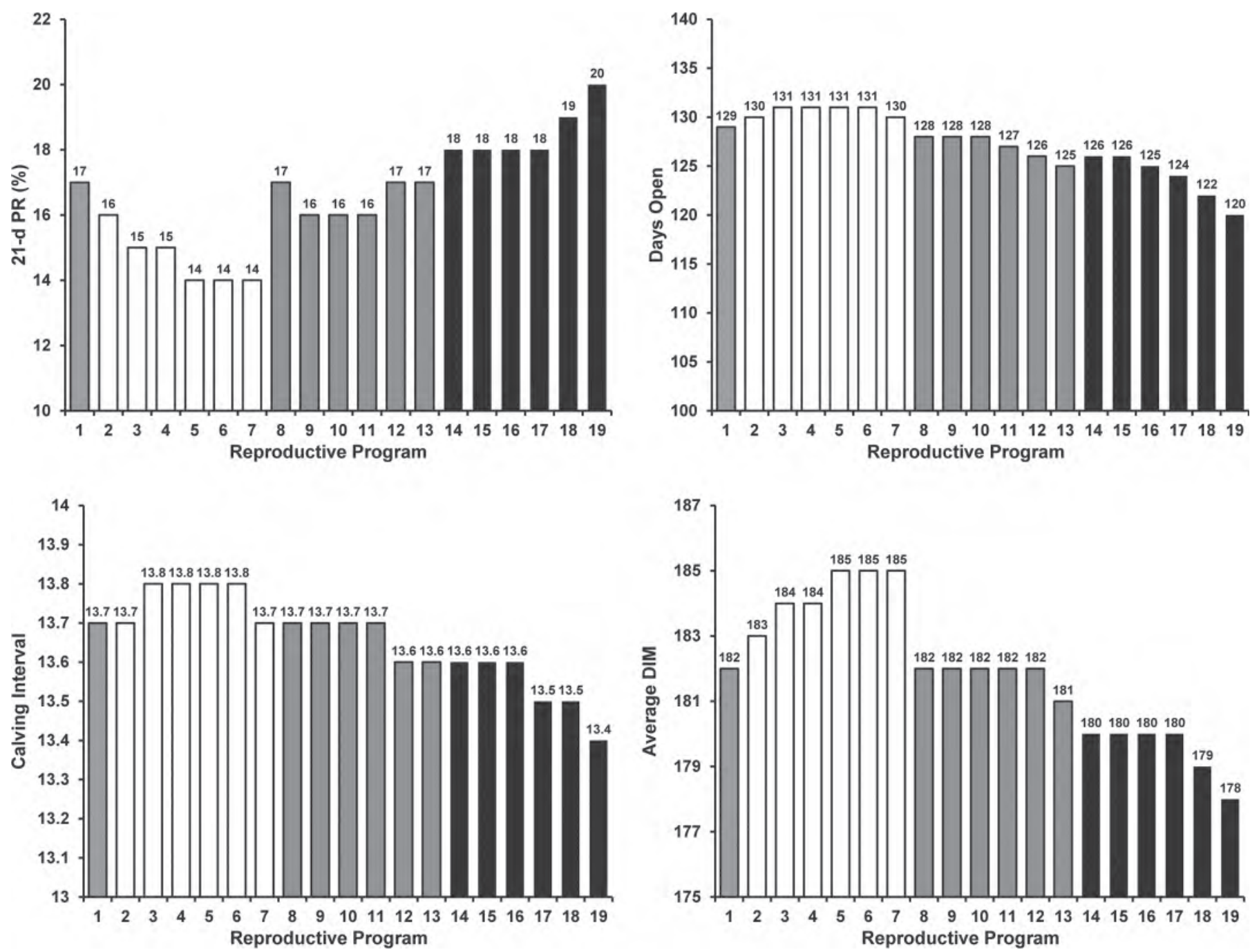

Figure 5. Reproductive parameters calculated by the daily Markov-chain model based on reproductive dynamics simulated with data for the case study. $\mathrm{PR}=$ pregnancy rate.

programs followed similar trends to the 21-d PR, some specific programs had slight discrepancies between 21-d PR and average DO. For example, program TAI+ED 2, which had a $16 \%$ 21-d PR, had either 2 or 3 additional DO than other combined programs with the same 21-d PR (programs 9 to 11). Conversely, combined programs 8 to 10, despite having different 21-d PR, had similar DO. Projected calving intervals ranged between 13.4 and 13.8 mo and herd average DIM between 178 and 185, following the same trends as 21-d PR and DO (Figure 5).

Survival curves for pregnancy for a subset of programs are presented in Figure 6 to illustrate the effect of adding ED with different fertility outcomes to a $100 \%$ TAI program. The programs represented in Figure 6 are those with $50 \%$ of cows inseminated after ED (TAI+ED 4, 10, and 16) within each of the 3 CR groups (25, 30, and 35\%) and also the survival curve for the $100 \%$ TAI program (TAI 1). All combined programs had a greater proportion of pregnant cows after the end of the VWP at 50 DIM to 72 DIM when cows receive first service in TAI 1 . Thereafter, TAI 1 outperformed TAI+ED 4 throughout the entire breeding period until the DIM cutoff for AI, which was set at 300 DIM. Conversely, TAI 1 and TAI+ED 10 had similar percentages of pregnant cows, except for some specific time points in which TAI+ED 10 had more pregnant cows (because cows in TAI 1 had not yet received their subsequent TAI). At the end of the period, TAI 1 had a slightly greater percentage of pregnant cows. Finally, when comparing the performance of TAI 1 and TAI+ED 16, the program using ED had a greater 


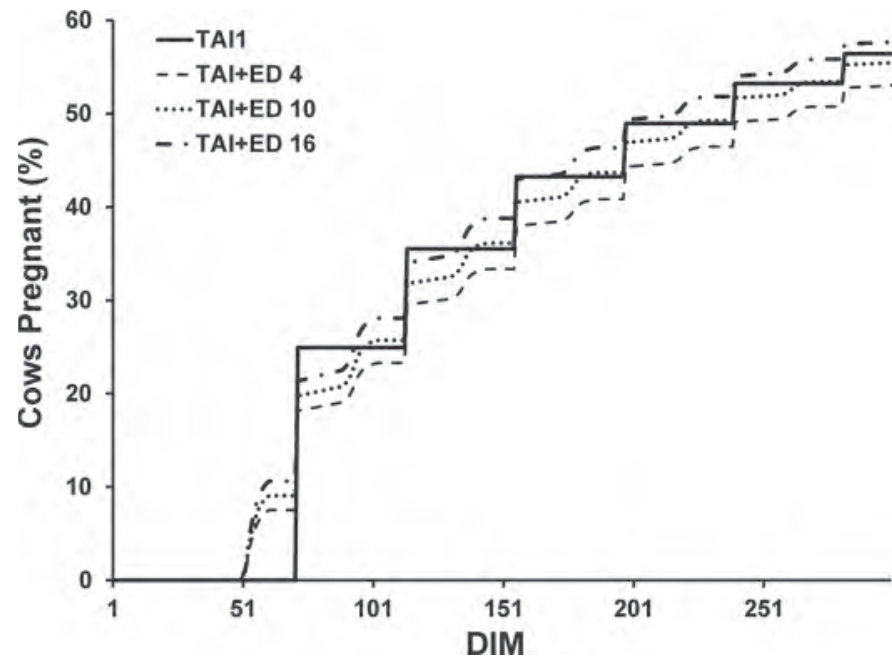

Figure 6. Accumulated percentage of pregnant cows until the DIM cutoff ( $300 \mathrm{~d}$ ) for insemination for 5 representative programs. Program timed AI (TAI) 1 was used as a baseline for comparison with the combined programs. Combined TAI + estrus detection (TAI+ED) programs 4, 10, and 16 were included to represent each one of the 3 groups with 25,30 , and $35 \%$ conception rate. All combined programs represented had $50 \%$ of cows inseminated after estrus detection. The values represented take into account the effect of replacement on the proportion of pregnant cows. percentage of pregnant cows throughout the entire AI period, except after each of the TAI services until 151 DIM.

\section{Matching Heifer Supply and Demand}

Finding a perfect balance between heifer replacement supply and demand by changing the DIM cutoff for AI services was possible for 11 out of the 19 programs (Table 3). All programs that presented a deficit of replacement heifers with the original 300 DIM cutoff required extra days for AI to reach a balance closest to zero. Conversely, for those programs with surplus heifers using a cutoff of 300 DIM, the closest balance to zero was obtained using a DIM cutoff of less than 300 d. The only exception was program TAI+ED 3, which had a perfect (zero) balance with the original 300 DIM cutoff.

For the 100\% TAI program (TAI 1) and each of the programs with 30 and $35 \%$ CR (TAI+ED 8 to 19), the NV increased when the supply and demand of heifers was balanced (Figure 7a). Conversely, for the combined programs with $25 \% \mathrm{CR}$ (TAI+ED 2 to 7 ), the NV (\$/ cow per year) improved slightly for TAI+ED 2, did not change for TAI+ED 3, and decreased in all the other

Table 3. Balance between heifer supply and demand after the initial simulation with the 300-DIM cutoff and after adjusting the DIM cutoff to match heifer supply and demand

\begin{tabular}{lccc}
\hline Program & $\begin{array}{c}\text { Heifer balance } \\
\text { 300 DIM (heifers) }\end{array}$ & $\begin{array}{c}\text { Heifer balance new } \\
\text { cutoff (heifers) }\end{array}$ & $\begin{array}{c}\text { DIM new } \\
\text { cutoff }\end{array}$ \\
\hline TAI 1 & 29.2 & -3.7 & 270 \\
TAI+ED 2 & 11.0 & 281 \\
TAI+ED 3 & 11.0 & 0.0 & 300 \\
TAI+ED 4 & 0.0 & 0.0 & 302 \\
TAI+ED 5 & -3.7 & 0.0 & 310 \\
TAI+ED 6 & -14.6 & 3.7 & 325 \\
TAI+ED 7 & -18.3 & 0.0 & 325 \\
TAI+ED 8 & -21.9 & 0.0 & 280 \\
TAI+ED 9 & 21.9 & 0.0 & 280 \\
TAI+ED 10 & 18.3 & 0.0 & 280 \\
TAI+ED 11 & 18.3 & -3.7 & 281 \\
TAI+ED 12 & 11.0 & 0.0 & 280 \\
TAI+ED 13 & 14.6 & 0.0 & 239 \\
TAI+ED 14 & 18.3 & 3.7 & 245 \\
TAI+ED 15 & 36.5 & 3.7 & 240 \\
TAI+ED 16 & 36.5 & 3.7 & 240 \\
TAI+ED 17 & 40.2 & 0.0 & 240 \\
TAI+ED 18 & 36.5 & 3.7 & 235 \\
TAI+ED 19 & 40.2 & 0.0 & \\
\hline
\end{tabular}

${ }^{1}$ Program timed AI (TAI) 1 relied on $100 \%$ TAI for all services after synchronization of ovulation for first postpartum AI with the Presynch-Ovsynch protocol, whereas the Ovsynch protocol was used for the second and subsequent AI services. The voluntary waiting period (VWP) was $72 \mathrm{~d}$ and the interval between 2 successive inseminations was $42 \mathrm{~d}$.

${ }^{2}$ Programs combining TAI with estrus detection (TAI+ED 2 to 19), used the same synchronization of ovulation protocols as TAI except that AI after ED was added between the end of the VWP set at 50 DIM and the first TAI at 72 DIM and in between TAI services (ED was discontinued at the time of the first GnRH injection of the Ovsynch protocol for all AI services). 

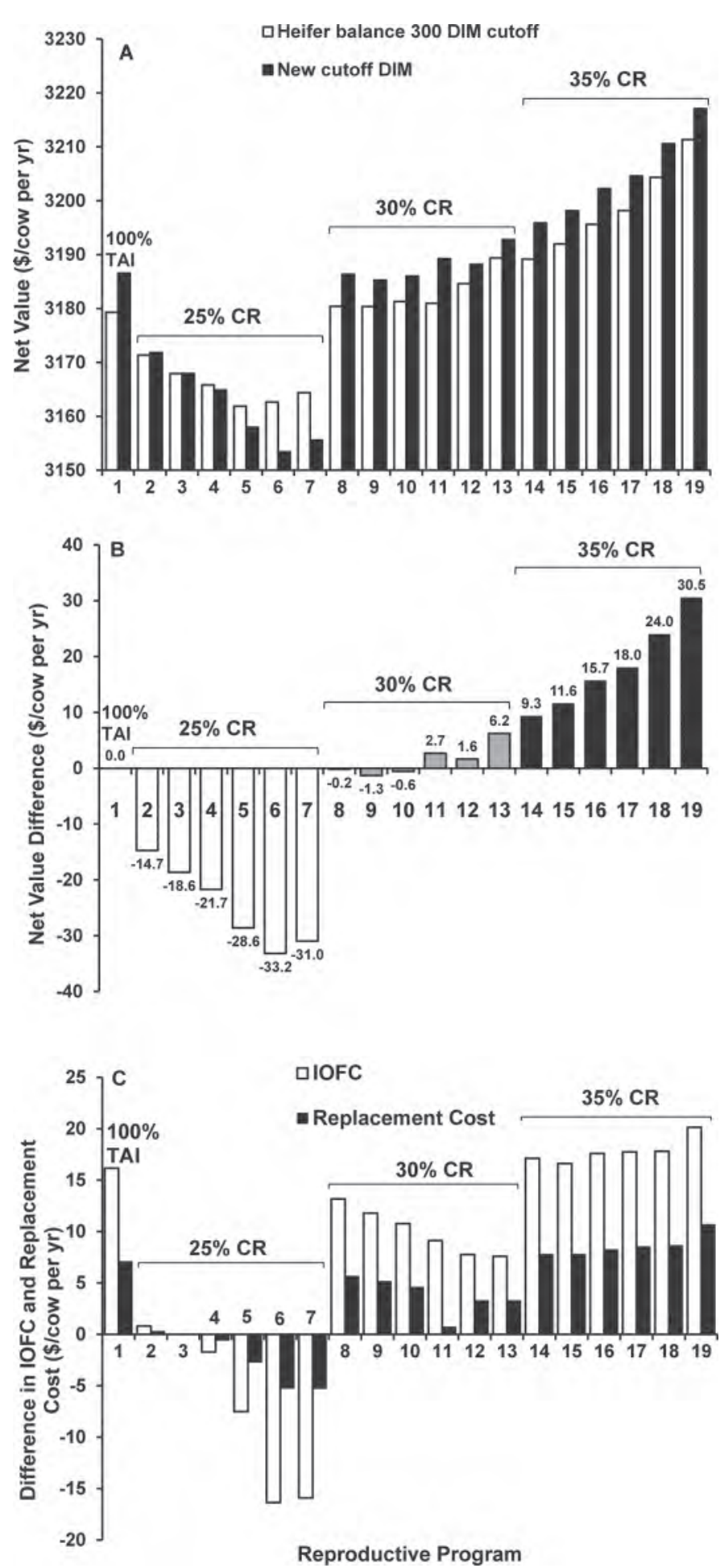

Figure 7. Changes in net value (NV; $\$$ /cow per year; panel A), difference between timed AI (TAI) and combined programs (panel B), and relative contribution of milk income over feed cost (IOFC) and replacement cost (panel C) to the NV after adjusting the DIM cutoff for insemination to match the replacement heifer supply and demand. $\mathrm{CR}=$ conception rate. programs with the major changes observed for those with equal or greater than $60 \%$ of cows receiving $\mathrm{AI}$ after a detected estrus (Figure 7A).

When comparing the difference between each of the combined programs and TAI 1, similar patterns were observed to when the DIM cutoff for AI was 300 , except for some of the TAI+ED programs with $30 \% \mathrm{CR}$, which shifted from a slightly positive to a slightly negative difference with the $100 \%$ TAI program (Figure 7B). Moreover, some significant changes were observed for the differences among programs. Program 1 outperformed combined programs with $25 \%$ CR by a greater difference than without the heifer balance and either decreased or increased the difference gap with the programs with $30 \% \mathrm{CR}$. When compared with those programs with $35 \% \mathrm{CR}$, the differences were also smaller than when no balance in the heifer supply and demand was attempted.

An assessment of the relative contribution of the NV components after balancing heifer supply and demand showed that the factor with the greatest change was IOFC, followed by replacement cost (Figure 7C). By contrast, calf value and reproductive program cost had less variation. The average change in calf value was $-\$ 1.19 \mathrm{cow} / \mathrm{yr}$ with a range of $-\$ 3.65$ to $\$ 1.79 \mathrm{cow} /$ $\mathrm{yr}$, whereas, for reproductive program cost, the average change was $\$ 0.07 \mathrm{cow} / \mathrm{yr}$, with a range of $-\$ 0.04$ to $\$ 0.51 \mathrm{cow} / \mathrm{yr}$.

\section{DISCUSSION}

\section{Economic and Reproductive Performance}

Results obtained under the conditions specified for this particular case study indicated that the fertility of cows inseminated after a detected estrus and the consequent change in the population of cows reaching the TAI services are critical factors that affect the economic and reproductive outcomes in herds combining TAI and ED. When compared with a program relying solely on TAI with achievable results in commercial dairy farms, those programs having $25 \%$ CR to the ED services had a lower NV at all levels of ED. This was an interesting finding because when these lower $\mathrm{CR}$ values are observed, either due to inaccurate ED or other factors on a farm, allowing more cows to complete the synchronization of ovulation protocol and receive a TAI is a more effective strategy than inseminating them at an earlier DIM. As previously suggested by Giordano et al. (2011), it appears that inseminating cows detected in estrus before completion of a synchronization protocol and obtaining low $\mathrm{CR}$ has a negative effect on the overall reproductive performance because cows that would have a greater chance of becoming pregnant after 
TAI are prevented from it and are subject to a longer time interval until they are reinseminated and have a chance to conceive.

The detriment of increasing the percentage of cows receiving AI after ED in programs with $25 \% \mathrm{CR}$ increased as the percentage of cows receiving AI after ED increased from 30 to $60 \%$. Nonetheless, this trend was reversed when 70 and $80 \%$ of cows received AI after ED, probably reflecting the 2-percentage-point decrease in the CR to TAI for resynchronized services that reduced the gap in $\mathrm{CR}$ between cows receiving $\mathrm{AI}$ after a detected estrus and cows receiving TAI. Obtaining very similar CR after ED or TAI services favored the insemination of cows in estrus because the probability of conception was similar to TAI, whereas the interbreeding interval became shorter.

As the CR to ED inseminations increased from 25 to $30 \%$, a major change was observed in the NV for all programs, which were superior to TAI 1 in all cases. The small differences observed between TAI 1 and programs TAI+ED 8 through 11 indicated no major economic advantage of performing either of these programs. Conversely, larger differences were observed for programs TAI+ED 12 and 13 that inseminated 70 and $80 \%$ of cows to a detected estrus. An interesting implication of these observations is that when the percentage of cows receiving AI after a detected estrus before firstservice TAI and subsequent AI services is within 30 to $60 \%$ with a CR of $30 \%$, neither major gains nor losses should be expected if cows receive AI after a detected estrus or reach the TAI. Even though it may be possible to inseminate up to 70 to $80 \%$ of cows in estrus between TAI services, these 2 scenarios are rarely seen in commercial dairy operations performing estrus detection by conventional means. Indeed, large field trials have shown that the percentage of cows receiving AI at estrus after presynchronization varies between 50 to 60\% (Stevenson and Phatak, 2005; Lima et al., 2009; Chebel and Santos, 2010).

When compared with the $100 \%$ TAI program and all other combinations of TAI and ED, programs having $35 \%$ CR had substantially greater NV at all levels of ED. These programs outperformed TAI 1 by $\$ 9.9$ to $\$ 32.0$ cow/yr and some of the lowest-performing programs by 40 to $\$ 50$ cow/yr. In all cases, when compared with TAI 1, these programs had a smaller percentage of pregnant cows up to approximately 150 DIM because the higher CR to first service in TAI 1 generated a substantial difference after each of the TAI services, particularly during the early part of the breeding period (data shown in Figure 6 for TAI+ED 16 only). However, as DIM progressed, the greater CR to the ED inseminations in the combined programs outweighed this early difference. Indeed, in all cases, combined programs had a greater percentage of pregnant cows by the DIM cutoff for AI. It was not surprising to observe better performance for the programs with $35 \%$ CR because for second and subsequent AI services, the CR was always greater than that of TAI breedings. Cows inseminated after a detected estrus had a shorter interbreeding interval and greater chances of conceiving than cows reaching the TAI.

A major practical implication of this study is that it identified some interesting trends in the economic and reproductive performance of programs typically used in commercial US dairy herds. According to our results, when 30 to $80 \%$ of cows receive AI to a detected estrus and the CR of these inseminations is $25 \%$, the economic outcome will be negative when compared with the $100 \%$ TAI program. Another factor to consider is the impact of the change in the population of cows reaching the TAI as the percentage of cows that receive AI after estrus in the combined programs increased. The consequent decrease in $\mathrm{CR}$ for cows reaching the TAI services was another reason for the overall lower economic performance of these programs. These observations suggest that allowing cows to complete the synchronization protocol and receive a TAI would be a preferred strategy. Conversely, whenever the CR to ED inseminations was 30 or $35 \%$, there was always an economic advantage when inseminating cows detected in estrus, even though a different magnitude of NV change was anticipated according to the percentage of cows receiving $\mathrm{AI}$ after $\mathrm{ED}$ and the resulting $\mathrm{CR}$.

\section{Simulated Herd Reproductive Dynamics}

As expected, widely different reproductive dynamics were observed among programs. Despite the lag time until first-service AI for cows on TAI 1, the greater CR resulted in greater (after first services in all cases) or equal percentages of pregnant cows after each of the TAI services. This advantage was maintained throughout most of the breeding period when compared with the inferior combined programs; however, for programs outperforming TAI 1 the major differences were observed only after 150 DIM.

The impact on reproductive dynamics of the specific probability distributions used to distribute the total proportion of cows AI in estrus is illustrated by the curvilinear shape of the survival curve to pregnancy between TAI services in Figure 6. Between the VWP and first service, and then for each time period between successive services the curves reflected the percentage of cows becoming pregnant after insemination after detection of estrus. 
The use of probability distributions to allocate cows receiving AI after a detected estrus is a novel capability of this model. This approach enables more precise modeling of the actual pattern of inseminations occurring on the farm when cows are submitted to synchronization of estrus or ovulation protocols (Bartolome et al., 2005; Chebel et al., 2006). From a reproductive and economic performance perspective, this modeling approach is beneficial because it allows for better evaluation of the benefits or disadvantages of inseminating cows detected in estrus.

The values for reproductive parameters calculated by the model based on the simulated herd dynamics are within the range of values typically observed in dairy herds using these types of programs. Therefore, within the constraints of mathematical simulations of biological events, the model calculations are reliable because they accurately reflect the dynamics of commercial herds.

Presenting the 21-d PR with a VWP of 50 DIM for TAI 1 when the actual VWP for this program was 72 DIM was done to compare all of the programs at a similar DIM after calving. Interestingly, the NV of TAI 1 was equal or very similar to that of combined programs having the same value for 21-d PR at 50 DIM (i.e., TAI+ED 8, 12, and 13). Conversely, an important difference in NV was observed between TAI+ED 19 and TAI 1, regardless of having the same 21-d PR, when the VWP for TAI 1 was set at 72 DIM. These are valuable observations, implying that manipulating the duration of the VWP when performing 21-d PR calculations may lead to the conclusion that a program with a greater 21-d PR is superior to others, when in fact, the economic outcome may be similar to or worse than that of the programs being compared. Thus, the VWP to compare the 21-d PR of the 19 programs was standardized at 50 DIM rather than adjusting it to the timing of the first insemination in TAI 1. Nevertheless, it is always important to consider these implications within the context of the case study and not extrapolate these conclusions to all herds and for all situations.

\section{Relative Contribution of Studied Economic Factors}

Under all circumstances, IOFC was the factor with the greatest positive contribution to the NV. When compared with the other factors, the contribution of IOFC to the NV was approximately 18 times greater than the replacement cost, 95 times greater than reproductive program cost, and 80 times greater than calf value. This was not surprising because the major source of income for dairy farms is milk sales (St-Pierre et al., 2000; White et al., 2002; De Vries, 2004). The second factor of importance was replacement cost, followed by the cost of performing the reproductive programs and the added value of newborn calves. Interestingly, similar observations were reported by Giordano et al. (2011).

In spite of the major contribution of IOFC to the total NV, this factor accounted for an average difference among programs of $\$ 7.8 /$ cow per year. Thus, reproductive efficiency of the compared programs had an effect on milk production, which in turn, accounted for most of the economic benefits in programs with superior economic performance. By contrast, the average and the range for differences among programs due to replacement cost and reproductive program cost was similar in magnitude and variation. Both factors had a significantly smaller contribution to the total NV, but in proportion had a very significant contribution to the differences among programs. The fact that these 2 components of the NV were much less significant than IOFC, but in some cases contributed to major differences between programs, suggests that the difference in profitability can be greatly affected by the impact of replacement cost and reproductive program cost.

Finally, calf value presented the least influence on the differences among programs. This could be explained, at least in part, by the low value of newborn calves and replacement heifers as well as the high value of replaced cows used as input in the case study.

\section{Matching Supply and Demand of Heifer Replacements}

Reproductive dynamics of a dairy herd affects the level of replacement (Pecsok et al., 1994; De Vries et al., 2010) and the stream of replacement heifers because of the difference in the number of calves born (Louca and Legates, 1968; Pecsok et al., 1994). Under most circumstances, dairy herds with excellent reproductive performance have an abundant supply of replacement heifers, creating an opportunity to increase replacement pressure of the least profitable cows if the herd size is to be maintained constant. In addition, another consequence of optimal reproductive performance is that lower levels of replacement due to reproductive failure will be observed. On the contrary, herds with suboptimal reproductive performance are likely to have deficits of replacement heifers and have greater levels of replacement due to reproductive failure. This notion, which is usually observed in commercial dairy operations, is supported by the results of the case study presented here and has been previously suggested by others (Plaizier et al., 1997). In this regard, adjusting the cutoff DIM for insemination in the simulated herd 
from the case study was a first step to determine the economic impact of having a balance closest to zero in the heifer supply and demand. The results of the initial simulations for inseminations clearly showed that programs having superior reproductive performance had a surplus of heifers, whereas programs with lower performance had a heifer deficit.

Balancing the heifer supply and demand affected the NV. Regardless of differences in the magnitude of the change, all programs that had a heifer surplus benefited. When all programs were compared again after simulations with the new cutoff, the most dramatic change was the greater difference observed between TAI 1 and the combined programs with $25 \%$ CR. The major reason for the change in $\mathrm{NV}$ was an increase in IOFC sufficiently high to offset the greater replacement cost observed in programs that had an improved NV.

It is widely accepted that maximum profitability is attained when cows spend the maximum amount of time in the most efficient part of their lactation curve when the marginal milk production over feed consumed is greatest (Pecsok et al., 1994; Ferguson and Galligan, 1999). In general, cows are most efficient during early and midlactation, with a major decrease in efficiency as lactation progresses (Britt et al., 2003). Therefore, changes in IOFC observed after running the new simulations to attain heifer balance were due to the changes in the herd dynamics resulting from the application of the new cutoffs. For programs with improved IOFC, cows that were still not pregnant during late lactation were forced to leave the herd and were replaced with a first-lactation cow. As a result, in those programs that shortened the cutoff for insemination, the average DIM for the herd decreased by 1 to $2 \mathrm{~d}$ and the population of cows shifted toward a younger herd with greater percentage of first-parity cows (data not shown). Interestingly, opposite trends were observed for those programs in which the cutoff for insemination was extended beyond $300 \mathrm{~d}$.

\section{Daily Markov-Chain Simulation Model}

Performing calculations on daily steps presents many advantages but certainly one of the most important is to allow performing very detailed calculations of reproductive events. Simulating the entire lifetime of cows is another interesting feature of this model because it is widely accepted that one of the major benefits of improved reproductive performance is to have cows pregnant early in the current lactation (up to a certain point) so that they begin their next lactation cycle earlier (Louca and Legates, 1968). Therefore, having multiple lactations simulated in the model allows cap- turing the benefits of early pregnancy in the current and subsequent lactations.

Having separate dimensions for nonpregnant and pregnant cows enables the model to simulate the events of gestation and those that apply only to nonpregnant cows in a separate and detailed manner. When cows undergo pregnancy loss they are not replaced but rather they have the opportunity to be reincorporated into the stream of nonpregnant cows and be reinseminated. In addition, the cost associated with the pregnancy diagnosis can be applied only to those cows that are actually being diagnosed.

The addition of the heifer module allowed adding a new dimension to the model calculations. Each reproductive management program has specific heifer demands according to the level of replacement and death that can be supplied or not by the stream of replacements. However, in those cases where the supply and demand were different, the model allowed balancing the supply of heifers to meet the needs of the herd under the conditions generated by its own dynamics.

Many other interesting additions make this daily Markov-chain model robust and comprehensive. Some of them include, but are not limited to, coding cows as do not breed and not replacing them immediately, calculation of BW throughout lactation, fitting of lactation curves by the MilkBot model (Ehrlich, 2011), and the calculation of reproductive parameters typically used on commercial dairy herds.

\section{CONCLUSIONS}

The results of the present study using a comprehensive daily Markov-chain simulation model capable of simulating dairy herd dynamics in a detailed manner indicated that the fertility of cows inseminated after a detected estrus and the consequent change in the population of cows reaching the TAI services in programs combining estrus detection and TAI are critical factors that affect the economic and reproductive outcomes in dairy herds. When compared with a program relying solely on TAI with achievable results in commercial dairy farms, programs having $25 \% \mathrm{CR}$ to the ED services had a lower NV at all levels of ED. Conversely, all programs having 30 and 35\% CR had greater NV than the $100 \%$ TAI program. In this case, the magnitude of the difference between the combined programs and the $100 \%$ TAI was proportional to the CR and percentage of cows AI after ED. Among the components of the NV, under all circumstances, IOFC was the factor with the greatest positive contribution to the NV and the differences in NV between programs. Replacement and reproductive program costs had less impact than 
IOFC, but greater than calf value. Balancing the heifer supply and demand by changing the DIM cutoff for insemination affected the NV of the programs simulated. Regardless of differences in the magnitude of the change, most of the programs, including the $100 \%$ TAI and those with 30 and $35 \%$ CR to ED inseminations, which had a heifer surplus, benefited. Conversely, most programs with $25 \% \mathrm{CR}$ to ED inseminations experienced a decrease in NV.

\section{ACKNOWLEDGMENTS}

This project was supported by Agriculture and Food Research Initiative Competitive Grant No. 2010-8512220612 from the US Department of Agriculture National Institute of Food and Agriculture (Washington, DC). This research was also supported by Hatch project WIS01171 to P. M. Fricke and Hatch project WIS01577 to V. E. Cabrera.

\section{REFERENCES}

AgSource Cooperative Services. 2011. DHI Benchmarks. Revised January 1, 2011. http://agsource.crinet.com/page849/DHIBenchmarks.

Bartolome, J. A., A. Sozzi, J. McHale, P. Melendez, A. C. M. Arteche, F. T. Silvestre, D. Kelbert, K. Swift, L. F. Archbald, and W. W. Thatcher. 2005. Resynchronization of ovulation and timed insemination in lactating dairy cows, II: Assigning protocols according to stages of the estrous cycle, or presence of ovarian cysts or anestrus. Theriogenology 63:1628-1642.

Bello, N. M., J. P. Steibel, and J. R. Pursley. 2006. Optimizing ovulation to first GnRH improved outcomes to each hormonal injection of Ovsynch in lactating dairy cows. J. Dairy Sci. 89:3413-3424.

Britt, J. H. 1985. Enhanced reproduction and its economic implications. J. Dairy Sci. 68:1585-1592.

Britt, J. S., R. C. Thomas, N. C. Speer, and M. B. Hall. 2003. Efficiency of converting nutrient dry matter to milk in Holstein herds. J. Dairy Sci. 86:3796-3801.

Cabrera, V. E. 2010. A large Markovian linear program to optimize replacement policies and dairy herd net income for diets and nitrogen excretion. J. Dairy Sci. 93:394-406.

Cabrera, V. E., A. de Vries, and P. E. Hildebrand. 2006. Prediction of nitrogen excretion in dairy farms located in north Florida: A comparison of three models. J. Dairy Sci. 89:1830-1841.

Cabrera, V. E., and J. O. Giordano. 2010. Economic decision making for reproduction. Pages 77-86 in Proc. Dairy Cattle Reproduction Council Convention, St. Paul, MN. Dairy Cattle Reproduction Council, Hartland, WI.

Caraviello, D. Z., K. A. Weigel, P. M. Fricke, M. C. Wiltbank, M. J. Florent, N. B. Cook, K. V. Nordlund, N. R. Zwald, and C. L. Rawson. 2006. Survey of management practices on reproductive performance of dairy cattle on large US commercial farms. J. Dairy Sci. 89:4723-4735.

Chebel, R. C., and J. E. Santos. 2010. Effect of inseminating cows in estrus following a presynchronization protocol on reproductive and lactation performances. J. Dairy Sci. 93:4632-4643.

Chebel, R. C., J. E. Santos, R. L. Cerri, H. M. Rutigliano, and R. G. Bruno. 2006. Reproduction in dairy cows following progesterone insert presynchronization and resynchronization protocols. J. Dairy Sci. 89:4205-4219.

Chebel, R. C., J. E. Santos, J. P. Reynolds, R. L. Cerri, S. O. Juchem, and M. Overton. 2004. Factors affecting conception rate after ar- tificial insemination and pregnancy loss in lactating dairy cows. Anim. Reprod. Sci. 84:239-255.

De Vries, A. 2004. Economics of delayed replacement when cow performance is seasonal. J. Dairy Sci. 87:2947-2958.

De Vries, A. 2006. Economic value of pregnancy in dairy cattle. J. Dairy Sci. 89:3876-3885.

De Vries, A., J. D. Olson, and P. J. Pinedo. 2010. Reproductive risk factors for culling and productive life in large dairy herds in the eastern United States between 2001 and 2006. J. Dairy Sci. 93:613-623.

Ehrlich, J. L. 2011. Quantifying shape of lactation curves, and benchmark curves for common dairy breeds and parities. Bovine Pract. 45:88-95.

Ettema, J. F., and J. E. Santos. 2004. Impact of age at calving on lactation, reproduction, health, and income in first-parity Holsteins on commercial farms. J. Dairy Sci. 87:2730-2742.

Ferguson, J. D., and D. T. Galligan. 1999. Veterinary reproductive programs. Pages 133-137 in Proc. 32nd Annual Convention of the American Association of Bovine Practitioners (AABP), Nashville, TN. AABP, Opelika, AL

Fetrow, J., D. McClary, R. Harman, K. Butcher, L. Weaver, E. Studer, J. Ehrlich, W. Etherington, W. Guterbock, D. Klingborg, J. Reneau, and N. Williamson. 1990. Calculating selected reproductive indices: Recommendations of the American Association of Bovine Practitioners. J. Dairy Sci. 73:78-90.

Fricke, P. M., D. Z. Caraviello, K. A. Weigel, and M. L. Welle. 2003. Fertility of dairy cows after resynchronization of ovulation at three intervals following first timed insemination. J. Dairy Sci. 86:3941-3950.

Giordano, J. O., P. M. Fricke, M. C. Wiltbank, and V. E. Cabrera. 2011. An economic decision-making support system for selection of reproductive management programs on dairy farms. J. Dairy Sci. 94:6216-6232.

Groenendaal, H., D. T. Galligan, and H. A. Mulder. 2004. An economic spreadsheet model to determine optimal breeding and replacement decisions for dairy cattle. J. Dairy Sci. 87:2146-2157.

Kalantari, A. S., H. Mehrabani-Yeganeh, M. Moradi, A. H. Sanders, and A. De Vries. 2010. Determining the optimum replacement policy for Holstein dairy herds in Iran. J. Dairy Sci. 93:2262-2270.

Keskin, A., G. Yilmazbas-Mecitoglu, E. Karakaya, A. Alkan, H. Okut, A. Gumen, and M. C. Wiltbank. 2011. Effect of presynchronization strategy prior to Ovsynch on fertility at first service in lactating dairy cows. J. Dairy Sci. 94(E-Suppl. 1):191. (Abstr.)

Kuhn, M. T., J. L. Hutchison, and G. R. Wiggans. 2006. Characterization of Holstein heifer fertility in the United States. J. Dairy Sci. 89:4907-4920.

Lima, J. R., F. A. Rivera, C. D. Narciso, R. Oliveira, R. C. Chebel, and J. E. Santos. 2009. Effect of increasing amounts of supplemental progesterone in a timed artificial insemination protocol on fertility of lactating dairy cows. J. Dairy Sci. 92:5436-5446.

Louca, A., and J. E. Legates. 1968. Production losses in dairy cattle due to days open. J. Dairy Sci. 51:573-583.

Meadows, C., P. J. Rajala-Schultz, and G. S. Frazer. 2005. A spreadsheet-based model demonstrating the nonuniform economic effects of varying reproductive performance in Ohio dairy herds. J. Dairy Sci. 88:1244-1254.

Moreira, F., C. Orlandi, C. A. Risco, R. Mattos, F. Lopes, and W. W. Thatcher. 2001. Effects of presynchronization and bovine somatotropin on pregnancy rates to a timed artificial insemination protocol in lactating dairy cows. J. Dairy Sci. 84:1646-1659.

NRC. 2001. Nutrient Requirements of Dairy Cattle. 7th ed. Natl. Acad. Sci., Washington, DC.

Olynk, N. J., and C. A. Wolf. 2008. Economic analysis of reproductive management strategies on US commercial dairy farms. J. Dairy Sci. 91:4082-4091.

Olynk, N. J., and C. A. Wolf. 2009. Stochastic economic analysis of dairy cattle artificial insemination reproductive management programs. J. Dairy Sci. 92:1290-1299.

Pecsok, S. R., M. L. McGilliard, and R. L. Nebel. 1994. Conception Rates. 2. Economic value of unit differences in percentages of sire conception rates. J. Dairy Sci. 77:3016-3021. 
Plaizier, J. C., G. J. King, J. C. Dekkers, and K. Lissemore. 1997. Estimation of economic values of indices for reproductive performance in dairy herds using computer simulation. J. Dairy Sci. 80:2775-2783

Press, W. H., S. A. Teukolsky, W. T. Vetterling, and B. P. Flannery 2007. Numerical Recipes: The Art of Scientific Computing. 3rd ed. Page 801. Cambridge University Press, New York, NY.

Pursley, J. R., M. R. Kosorok, and M. C. Wiltbank. 1997. Reproductive management of lactating dairy cows using synchronization of ovulation. J. Dairy Sci. 80:301-306.

Pursley, J. R., M. O. Mee, and M. C. Wiltbank. 1995. Synchronization of ovulation in dairy cows using $\mathrm{PGF}_{2 \alpha}$ and GnRH. Theriogenology 44:915-923.

Rabiee, A. R., I. J. Lean, and M. A. Stevenson. 2005. Efficacy of Ovsynch program on reproductive performance in dairy cattle: A meta-analysis. J. Dairy Sci. 88:2754-2770.

Schmidt, G. H. 1989. Effect of length of calving intervals on income over feed and variable costs. J. Dairy Sci. 72:1605-1611.

Silva del Río, N., S. Stewart, P. Rapnicki, Y. M. Chang, and P. M. Fricke. 2007. An observational analysis of twin births, calf sex ratio, and calf mortality in Holstein dairy cattle. J. Dairy Sci. 90:1255-1264.

Souza, A. H., H. Ayres, R. M. Ferreira, and M. C. Wiltbank. 2008. A new presynchronization system (Double-Ovsynch) increases fertil- ity at first postpartum timed AI in lactating dairy cows. Theriogenology 70:208-215.

St-Pierre, N. R., and L. R. Jones. 2001. Forecasting herd structure and milk production for production risk management. J. Dairy Sci. 84:1805-1813

St-Pierre, N. R., D. Shoemaker, and L. R. Jones. 2000. The next $\$ 120,000$ : A case study to illustrate analysis of alternative farm investments in fixed assets. J. Dairy Sci. 83:1159-1169.

Stevenson, J. S., and A. P. Phatak. 2005. Inseminations at estrus induced by presynchronization before application of synchronized estrus and ovulation. J. Dairy Sci. 88:399-405.

USDA (US Department of Agriculture). 2010. Dairy 2007: Heifer Calf Health and Management. Practices on US Dairy Operations, 2007. No. 550.0110. USDA:Animal and Plant Health Inspection Service:Veterinary Services (USDA:APHIS:VS), Centers for Epidemiology and Animal Health (CEAH), Fort Collins, CO.

van Arendonk, J. A. M. 1985. A model to estimate the performance, revenues and costs of dairy cows under different production and price situations. Agric. Syst. 16:157-189.

White, S. L. G. A. Benson, S. P. Washburn, and J. T. Green Jr. 2002. Milk production and economic measures in confinement or pasture systems using seasonally calved Holstein and Jersey cows. J. Dairy Sci. 85:95-104. 